\title{
DISCUSSING THE EFFECT OF GAMMA PRIME COARSENING ON HIGH TEMPERATURE LOW STRESS CREEP DEFORMATION WITH RESPECT TO THE ROLE OF REFRACTORY ELEMENTS
}

\author{
T. Goehler ${ }^{1}$, C. Schwalbe ${ }^{2}$, J. Svoboda ${ }^{3}$, E. Affeldt ${ }^{1}$, R.F. Singer ${ }^{4}$ \\ ${ }^{1}$ MTU Aero Engines AG, Dachauer Straße 665, 80995 München, Germany \\ ${ }^{2}$ Cambridge University, Department of Material Science and Metallurgy, CB3 0FS Cambridge, United Kingdom \\ ${ }^{3}$ Academy of Sciences of the Czech Republic, Institute of Physics of Materials, 61662 Brno, Czech Republic \\ ${ }^{4}$ University of Erlangen, Institute of Science and Technology of Metals, 91058 Erlangen, Germany
}

Keywords: Coarsening, Solid-solution hardening, $\gamma / \gamma^{\prime}$-Thickness ratio, CALPHAD, Creep modelling

\begin{abstract}
Using the predictions made by a microstructure sensitive creep model for high- $\gamma^{\prime}$-containing superalloys, this study will compare the predictions made for the dislocation activity during different stages of the microstructural evolution against experimental results in order to understand the role and influence of microstructural coarsening. Furthermore a composition simulation was undertaken to present optimization potential with respect to microstructural coarsening properties.
\end{abstract}

\section{Introduction}

Ni-base SX-Superalloys are the state of art materials currently used in the high pressure and first stages of the low pressure turbine. Since their introduction to the turbine, continuous improvements to the production process and optimized alloy compositions have resulted in significantly extended creep lifetimes [7]. To further optimize alloy compositions according to this design criteria, physical based creep models have helped to speed up and reduce the costs of the design process. Additionally, these models can predict a number of key creep parameters for established compositions with a high level of accuracy.

\section{Experimental Procedure}

For this study, five commercial Ni-SX-Superalloys were selected, that display different rhenium to tungsten alloying ratios (see at.- $\%$ Table 1). Samples were cast and selected with a maximum offset of $\pm 15^{\circ}$ from the $<001>$ direction, and ultimately heattreated according to the alloy specific standard commercial cycles by Precision Castparts Cooperation. Specimens were thereafter polished at MTU.

Subsequently, creep experiments for all selected alloys were performed at $1050{ }^{\circ} \mathrm{C}$ and $150 \mathrm{MPa}$ interrupted after $0.2 \%, 0.5 \%$ $1 \%$ and $2 \%$ plastic strain. Samples were cut parallel to the tensile axis with a (110) plane normal and prepared according to relevant standards [1] to analyse the microstructural evolution using Zeiss Evo $60 \mathrm{SEM}$ at $20 \mathrm{kV}$. The images were taken in the dendritic cores of the samples at $5 \mathrm{k}$ and $10 \mathrm{k}$ magnification using a 50:50 signal ratio from the SE and BSE detector. Thereafter image analysis to quantify the change in matrix width on each interrupted test was performed, using the Software Imageaccess 12.

\section{Modelling Approaches}

The creep model published by Svoboda and Lukas [2] describes high temperature creep mechanisms for a uniaxial creep deformation in $<001>$-direction concisely and has been verified using experimental results of the alloy CMSX-4. The model is based upon an explicitly defined unit cell of one $\gamma^{\prime}$-particle surrounded by $\gamma$-matrix (Figure 1 ).

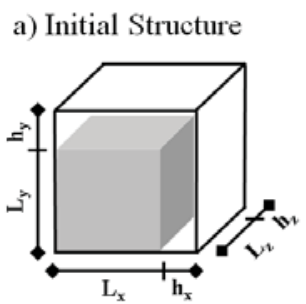

c) $0.5 \%-\mathrm{pl}$.

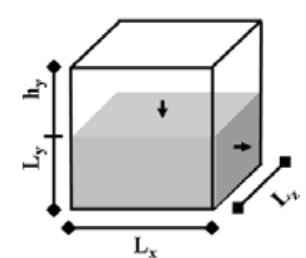

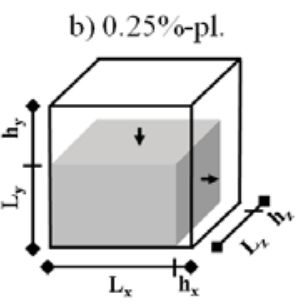

d) $2 \%-\mathrm{pl}$.

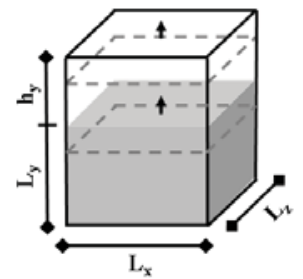

Figure 1. Unit cell as defined by Svoboda (initial structure) and gradual evolution assumed by the authors under a uniaxial creep deformation in $<001>$-direction. Arrows indicate the direction of interface migration between previous and actual step. a) initial cubic microstructure with 60 vol.-\%. $\gamma^{\prime}$-phase; b) transformation to raft like $\gamma / \gamma^{\prime}$-structure; c) completion of rafting by closure of vertical channels; d) continuous growth of unit cell in y-direction due to coarsening of the rafted structure.

The crystal plasticity and the microstructural evolution of the unit cell was calculated, using the models by Svoboda and Lukas as published in [2-6], which take following mechanisms into account:

1. Calculation of the internal stress state in each segment of the unit cell ( $\mathrm{x}-, \mathrm{y}-, \mathrm{z}$-matrix channel and precipitate) under consideration of the misfit.

2. Slip and multiplication of dislocations in discrete slip systems of the $\gamma$-matrix.

3. Cutting of the $\gamma^{\prime}$-particle. The dislocations generated in the matrix penetrate the $\gamma / \gamma^{\prime}$-interfaces. 
Table 1: Element composition of the studied alloys [wt.-/at.-\%]; balanced with nickel.

\begin{tabular}{|c|c|c|c|c|c|c|c|c|c|c|c|c|}
\hline \multicolumn{2}{|c|}{ Alloy } & Al & Co & $\mathrm{Cr}$ & Hf & Mo & $\operatorname{Re}$ & $\mathbf{T a}$ & $\mathbf{T i}$ & $\mathbf{W}$ & $\mathbf{N i}$ & Reference \\
\hline \multirow{2}{*}{ M247LC-SX } & at.- $\%$ & 12.3 & 10.2 & 9.3 & 0.5 & 0.4 & - & 1.0 & 1.3 & 3.3 & Bal. & \multirow{2}{*}{-} \\
\hline & wt.- $\%$ & 5.5 & 10.0 & 8.0 & 1.5 & 0.6 & - & 3.0 & 1.0 & 10.1 & Bal. & \\
\hline \multirow{2}{*}{ CMSX-6 } & at.- $\%$ & 9.9 & 4.7 & 10.7 & 0.03 & 1.7 & - & 0.6 & 5.5 & - & Bal. & \multirow{2}{*}[7]{} \\
\hline & wt.- $\%$ & 4.8 & 5.0 & 10.0 & 0.1 & 3.0 & - & 2.0 & 4.7 & - & Bal. & \\
\hline \multirow{2}{*}{ LEK94 } & at.- $\%$ & 13.8 & 7.3 & 6.7 & - & 1.2 & 0.8 & 0.7 & 1.2 & 1.1 & Bal. & \multirow{2}{*}{ [8] } \\
\hline & wt.- $\%$ & 6.5 & 7.5 & 6.1 & - & 2.0 & 2.5 & 2.2 & 1.0 & 3.5 & Bal. & \\
\hline \multirow{2}{*}{ ReneN5 } & at. $-\%$ & 13.8 & 7.7 & 8.1 & 0.06 & 0.9 & 1.0 & 2.3 & - & 1.6 & Bal. & \multirow{2}{*}{ [7] } \\
\hline & wt.- $\%$ & 6.2 & 8.0 & 7.0 & 0.2 & 2.0 & 3.0 & 5.8 & - & 5.0 & Bal. & \\
\hline \multirow{2}{*}{ PWA1484 } & at.- $\%$ & 13.0 & 10.5 & 6.0 & 0.03 & 1.2 & 1.0 & 1.9 & - & 2.1 & Bal. & \multirow{2}{*}[7]{} \\
\hline & wt.- $\%$ & 5.6 & 10.0 & 5.0 & 0.1 & 2.0 & 3.0 & 9.0 & - & 6.0 & Bal. & \\
\hline
\end{tabular}

4. Dynamic recovery of the dislocation structure. The dislocation loops spanning around the $\gamma^{\prime}$-particles move by the combination of slip and climb along the $\gamma / \gamma^{\prime}$-interfaces.

5. Morphological changes of the $\gamma^{\prime}$-precipitates by migration of $\gamma / \gamma^{\prime}$-interfaces related with diffusion transport of elements.

6. Coarsening of the rafted structure.

An initial study with the published creep model and corresponding data revealed, that the dimensions of the resulting unit cell evolve from $0.5 \mu \mathrm{m}$ up to $2 \mu \mathrm{m}$ and thus that the simulated microstructural evolution due to rafting and coarsening seems to be significantly overestimated. Therefore, channel widths and $\gamma^{\prime}$-volume fractions were obtained experimentally for all tested alloys after the interrupted creep tests at $1050{ }^{\circ} \mathrm{C}$ and $150 \mathrm{MPa}$ and used for the correction of the microstructural evolution. The particle widths were estimated by assuming a classical unit cell of average dimensions, which rafts towards an ideal 'sandwich' structure until two adjacent unit-cell particles coalesce subsequently allowing no further coarsening in any direction other than the direction of the load. (Figure 1).

With the closure of vertical channels, recovery mechanisms by dislocation climb become inactive. As a result, the deformation potential during different stages of creep life are principally based upon the dislocation glide and shearing ability in the system. In order to study these two dislocation mechanisms exclusively in this adaption of the model, dislocation climb was subsequently deactivated. Furthermore, the resistance to dislocation shearing was adapted to account for the case of strongly coupled dislocations as published in [7].

The coarsening parameter originally defined by Svoboda was replaced by the effective interdiffusion parameter $(\widetilde{D})$ as defined in [9], calculated using input data as mobility $\left(X_{i}^{\gamma}\right)$ and relative distribution of each element in the matrix phase $\left(D_{i}^{\gamma}\right)$ from the later described CALPHAD (Calculation of Phase Diagrams) approach. Furthermore, the lattice misfit was estimated using the alloy sensitive description of Vegards coefficients [10].

$$
\widetilde{D}=\frac{1}{\sum X_{i}^{\gamma} / D_{i}^{\gamma}}
$$

In order to find alloy compositions that can better respond to the microstructural improvement potential estimated by using crystal plasticity modelling, a CALPHAD [11] approach was applied. In the context of this study these calculations were performed using the software MatCalc ${ }^{\mathbb{B}}$ (version 5.61., MatCalc Engineering
$\mathrm{GmbH}$, Vienna, Austria) along with the relevant databases mc_ni_s03.tdb und mc_ni_v2.0.ddb [12].

The CALPHAD analysis centers around the variable $\widetilde{D}$ - the effective interdiffusion coefficient based on [9] as the main parameter determining the coarsening dynamics of the alloys studied [13]. Prior to the analysis a statistical based pre-selection of interactions, interdependencies and influential parameter was undertaken, using a linear regression model based on [14]. In the eight alloy element systems this resulted in 256 nodes which were then automatically analysed by coupling MatCalc ${ }^{\mathbb{R}}$ and MATLAB ${ }^{\circledR}$ (version 2011b, Mathworks GmbH, Ismaning, Germany).

\section{Results}

The plastic deformations measured over the tensile tests are plotted as datapoints in Figure 2 for the two first-generation (Re-free) and three second generation superalloys (up to 1 at.- $\%$ Re). Under the given test conditions the 1 at.- $\%$ rhenium containing alloys PWA1484 and RenéN5 exhibit an eight-fold increase in lifetime, when compared to the rhenium-free M247LC-SX and CMSX-6. The alloy LEK94 (0.8 at.-\% Re) displays a twofold improvement in lifetime compared to the rhenium-free alloys.

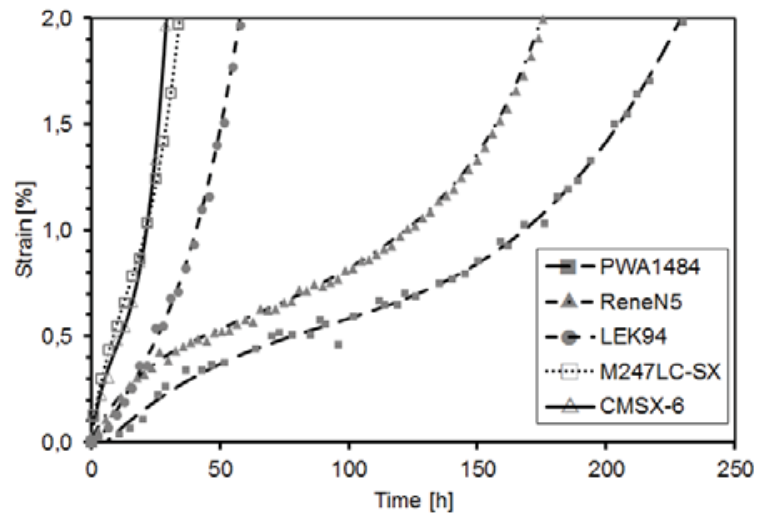

Figure 2. Measured creep strain vs experimental time for the tested alloys. Datapoints indicate raw data, lines a smoothened progression.

The test results from Figure 2 were smoothed and subsequently used to calculate the strain rate vs strain curves plotted in Figure 3. In Figure 3 the results display a reduced strain rate until about $0.5 \%$ plastic strain is accumulated in the sample, thereafter the strain rate increases continuously. At the local minimum, the strain rate differs between alloys of the first and second generation 
by about one order of magnitude. The time until the local strain rate minimum is consistently shorter than the time period with an increasing strain rate (compare Figures 2 and 3). The factor between both times varies by a factor of $1: 2$ to $1: 3$ when interrupting the creep tests at $2 \%$ plastic strain.

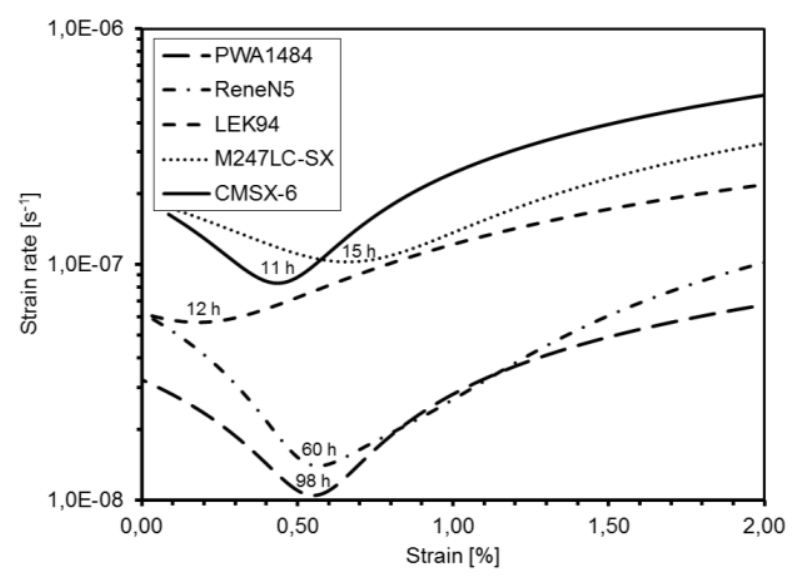

Figure 3. Strain rate vs. measured strain for the tested alloys.

These test results can be explained by studying the microstructural evolution in the samples qualitatively and quantitatively. Figure 4 shows an excerpt of the study, displaying the observed microstructures of the alloys M247LC-SX and RenéN5 deformed under test conditions and interrupted at the displayed strain. With $0.5 \%$ plastic stain accumulated, the microstructures have predominantly transformed to continuous horizontal rafts. This trend is visible even in M247LC-SX, despite the short testing time the number of vertical channels is very small and continuously decreases with test time. The rafted microstructure remains stable throughout the test regime (up to $2 \%$ plastic strain) and no topological inversion has been observed, instead a gradual isotropic coarsening of $\gamma^{\prime}$ rafts can be observed. This gradual coarsening can be quantified for different test times, by measuring the average distance in load direction between two $\gamma^{\prime}$-precipitates in the SEM micrographs.

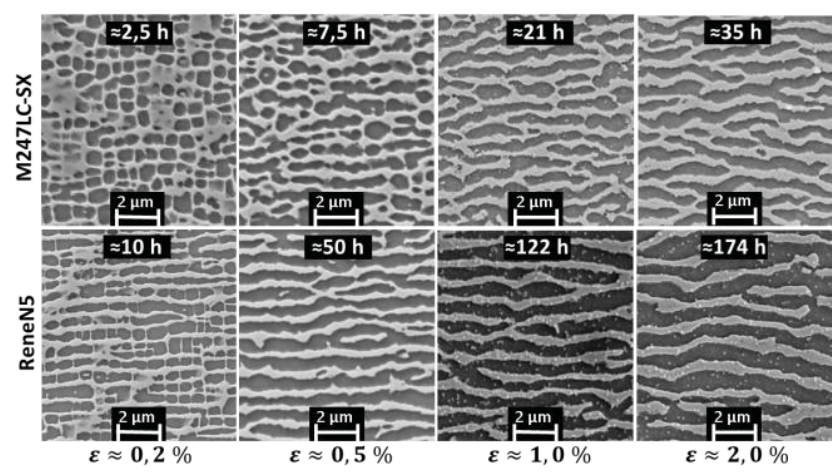

Figure 4. Microstructural evolution across interrupted creep tests until $0.2 \%, 0.5 \%, 1 \%$ and $2 \%$ plastic strain for the alloys ReneN5 and M247LC-SX. In bright is the $\gamma$-matrix, etched $\gamma^{\prime}$-regions remain dark.

Each datapoint for a specific test time in Figure 5 corresponds to the mean value from measurements taken in three different regions of at least $150 \mu \mathrm{m}^{2}$ area. The volume fraction of $\gamma^{\prime}\left(f_{\gamma^{\prime}}\right)$ for the alloys tested at room temperature was about 60 vol.- $\%$. In accordance with Ostwald ripening, the precipitate coarsening rate initially increases rapidly, then gradually slows down. Due to competitive growth processes, the standard deviation of measured mean $\gamma$-channel thickness increases from initially ca. $0.05 \mu \mathrm{m}$ to ultimately $0.1 \mu \mathrm{m}$ for all tested alloys. This means, that simultaneously with an increase in channel width an overall increase in microstructural inhomogeneity takes place. The alloy specific coarsening rates are shown in Figure 5, for first generation alloys they are significantly above the rates observed in the second generation alloys, closely resembling the alloy trend observed in Figure 2. In conclusion, these results imply, that under the test conditions a correlation exists between the coarsening kinetics and the creep strength.

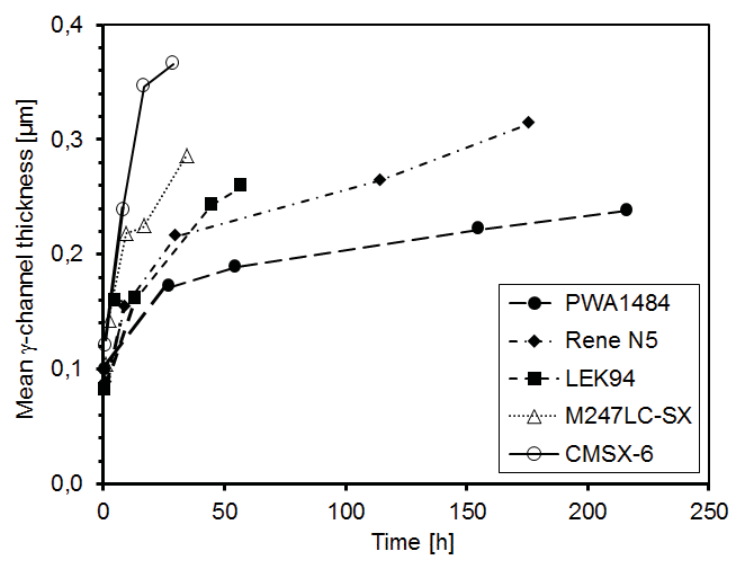

Figure 5. Evolution of the average $\gamma$-channel size in load direction under test conditions as a function of creep test time.

Feeding the microstructural results into the adapted creep model allows us to estimate the dislocation activities and mechanisms in order to form a hypothesis that could explain the observed correlation between creep strength and differences in the kinetics of microstructural evolution. As shown in Figure 1, the adapted model uses the measured microstructure dimensions to estimate the average $\gamma / \gamma^{\prime}$-dimensions in the unit cell. In the following, representative results using only model parameters based upon ReneN5 are displayed (a complete list of input parameters is stated in [13], whilst the remaining, qualitatively similar results can be found in [15].

The creep model is then used to estimate the time during which the microstructure has the potential to facilitate local $\gamma^{\prime}$-shearing by dislocations. This point is reached, when the shear stress within the $\gamma^{\prime}$-particles $\left(\tau_{P}\right)$ reaches the critical resolved shear stress $\left(\tau_{P K 0}\right) \cdot \tau_{P K 0}$ is defined by eq. (2) according to [7], where $\mathrm{b}$ is the burgers vector, $G$ the shear modulus, $w$ a material specific constant and $\gamma_{\mathrm{APB}}$ the antiphase boundary energy. The particle radius is substituted by $\mathrm{L}_{\mathrm{y}}$.

$$
\tau_{P K 0}=\sqrt{\frac{3}{2}}\left(\frac{G b}{L_{y}}\right) \sqrt{f_{\gamma^{\prime}}} \frac{w}{\sqrt{\pi^{3}}}\left(\frac{2 \pi \gamma_{A P B} L_{y}}{w G b^{2}}-1\right)^{1 / 2}
$$

The model however does not give an indication as to how substantial the additional plastic deformation by shearing the precipitate phases is. The primary carrier of deformation are (with the gradual completion of rafting) the horizontal $\gamma$-channels, where the critical shear stress $\left(\tau_{y 0}\right)$ and resolved shear stress $\left(\tau_{y}\right)$ decrease with rafting due to the effect of increasing channel width 
(less Orowan-resistance) and a relaxation of lattice misfit (due to deposited interfacial dislocations), see Figure 6. The misfit stresses add to the initial shear stress and increase it from $75 \mathrm{MPa}$ in a misfit free domain to above $120 \mathrm{MPa}$ under the given parameter conditions.

The 12 glide systems of the cubic face centered crystal structure can be simplified when applying a stress in $<001>$-direction to three glide system groups, each containing four equivalent slip systems. Only two of them are activated $\left(\tau_{y 1}, \tau_{y 3}\right)$.

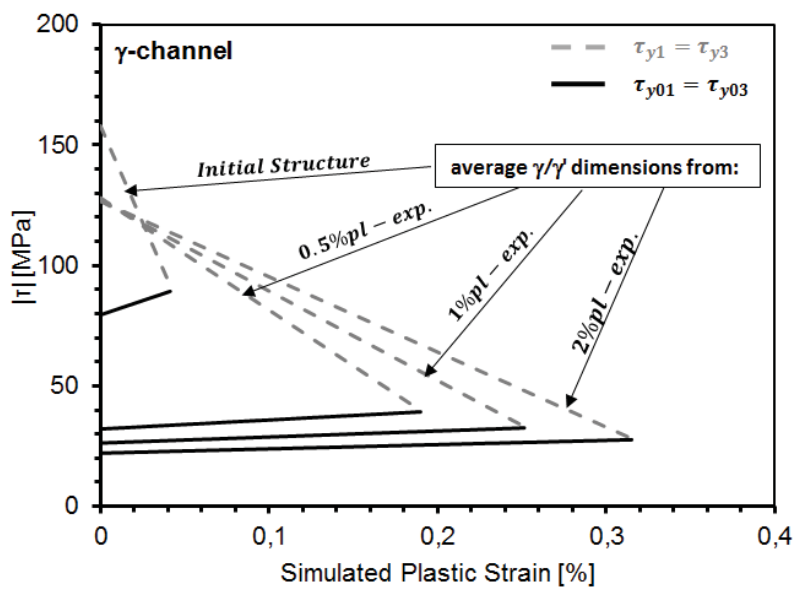

Figure 6. Evolution of the shear stress in the horizontal matrix channels for the averaged experimental microstructure dimensions.

Figure 7 depicts the evolution of the shear stress within $\gamma^{\prime}$-particles of average dimensions. The resolved shear stress $\left(\tau_{P 1}, \tau_{P 3}\right)$ lies significantly below the critical shear stress $\left(\tau_{P K 0}\right)$ required for particle shearing, thereby indicating that no plastic deformation can occur within a particle of average dimensions. Therefore, as a result of the assumptions made in the proposed model, all plastic deformation simulated will exclusively occur in the $\gamma$-matrix.

Crucially however, this method results in a significant discrepancy between the total amount of plastic strain determined experimentally and modelled (see Figures 6 and 7), with the simulated strain more than half an order of magnitude below the experimental. This difference can be attributed in part to the absence of dislocation climb and in part to the description of particle shearing. In this paper the latter context is investigated.

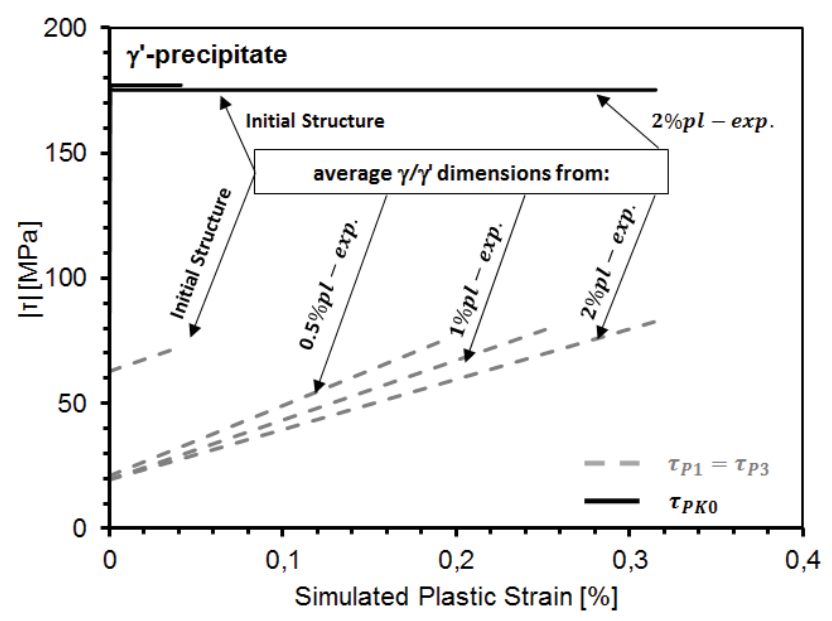

Figure 7. Evolution of the shear stress in the precipitates for the averaged experimental microstructure dimensions.

The shear stress required for a dislocation to enter a particle and its dependence from precipitate properties has been extensively studied $[7,16]$. Its dependence from particle size can be simulated and verified by using different particle to matrix thickness ratios to calculate their deformation potentials (see Figure 8). The particle to matrix thickness ratio is described by the parameter $\left(\varphi_{\gamma / \gamma}\right)$, that takes a value of $\varphi_{\gamma / \gamma^{\prime}}=0,64$ in the case of an average microstructure of ReneN5 at 2\% plastic deformation in described creep tests.

$$
\varphi_{\gamma / \gamma^{\prime}}=\frac{h_{y}}{L_{y}}
$$

Locally larger values of $\varphi_{\gamma / \gamma}$, will raise the resolved shear stress within the $\gamma^{\prime}$-particle, eventually exceeding the critical shear stress required for shearing (see Figure 8). For ReneN5, a value of $\varphi_{\gamma / \gamma^{\prime}}=2.5$ can be identified as critical thickness ratio for the local activation of shearing. Such ratios occur e.g. where a wide horizontal $\gamma$-channel is in contact with a shrinking $\gamma^{\prime}$-particle (see Figure 9). A smaller factor of $\varphi_{\gamma / \gamma^{\prime}}<0,64$ on the other hand, will not resolve in the activation of additional deformation mechanisms.

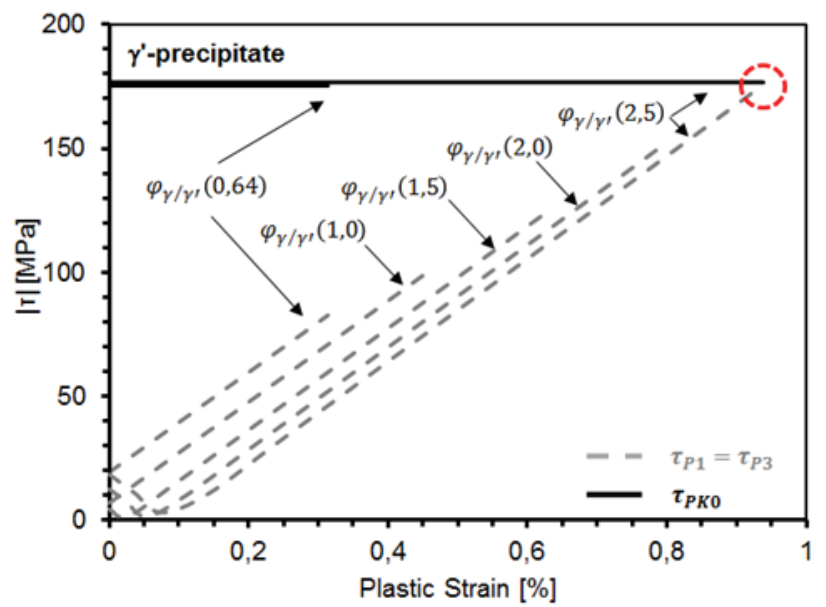

Figure 8. Critical resolved shear stress in the $\gamma^{\prime}$-particle for the labelled thickness ratios $\left(\varphi_{\gamma / \gamma}\right)$. 
Using the calculated critical thickness ratio, areas within the microstructure can then be detected where a dislocation line would have sufficient energy for particle shearing (Figure 9b-c). In the three dimensional strongly rafted structure (see focused ion beam tomography results [17]) the sites of a high thickness ratio appear to be fewer but nevertheless present. In conclusion, further $3 \mathrm{D}$ tomographs would help to study the areas of critical thickness ratio $\left(\varphi_{\gamma / \gamma},>2.5\right)$ and the evolution of frequency during creep testing.

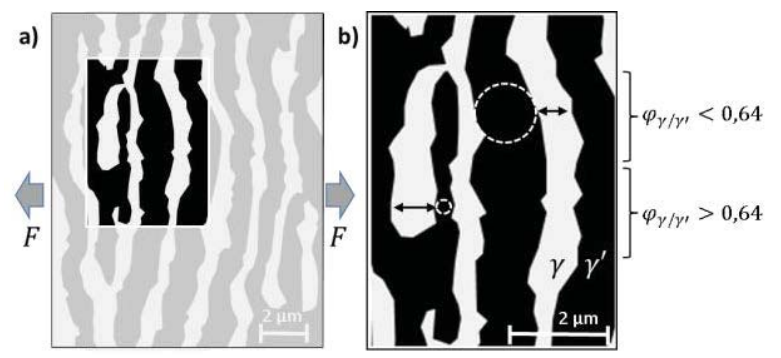

c)

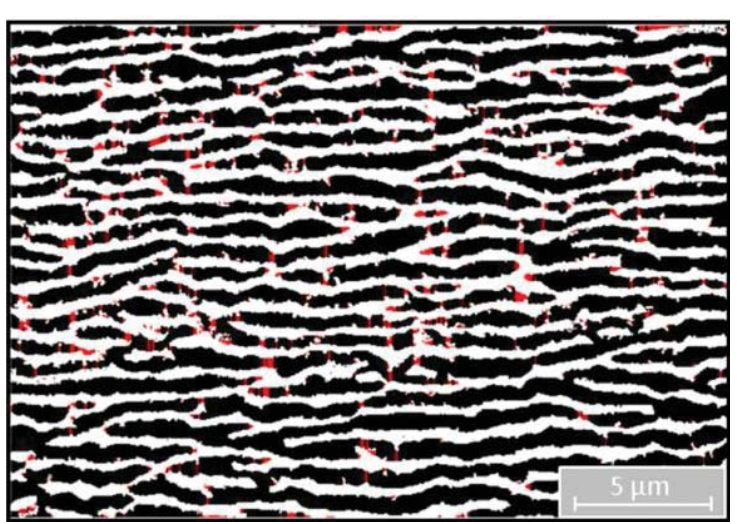

Figure 9. a) SEM-micrograph from the dendritic core of a rafted ReneN5 sample deformed until 2\% plastic strain. b) Illustration of the inherent microstructural variation between raft and channel sizes summarised by the factor $\left(\varphi_{\gamma / \gamma^{\prime}}\right)$. c) Implementation of the thickness ratio analysis on a micrograph from the identical material in a), highlighting areas where a critical thickness ratio of $\varphi_{\gamma / \gamma}=2.5$ has been exceeded.

Nevertheless, the calculations so far highlight the importance of microstructural homogeneity in terms of $\gamma^{\prime}$-raft thickness as an important design criterion. Due to competitive growth process, this homogeneity is dependent upon the level of coarsening, which in turn is driven by the effective diffusivity of alloying elements $[13,18,19]$. Therefore, the effective diffusivities within the tested alloys were modelled.

Table 2 displays the simulated $\widetilde{D}$ values and the partitioning coefficients for two representative elements tungsten and rhenium, the latter display a reasonable correlation with the experimental data. For validation purposes the diffusion parameters for each element are compared to experimental results at $1200{ }^{\circ} \mathrm{C}$ (the temperature where only the $\gamma$-Matrix remains) in Table 3 . The results indicate, that only rhenium and tungsten have a lower diffusivity than nickel and are thus the principal element additions that retard interdiffusion. The efficacy of their retardation potential is proportional to their $\gamma$-partitioning (see eq. 1), which highlights the exceptional combination of properties rhenium adds to the alloy.
Figure 10 then demonstrates, that the effective diffusivities can be correlated exponentially to the creep life until $2 \%$ pl. strain. Decreasing the effective interdiffusion coefficient then has the potential to enhance creep lifetime.

Table 2: Partitioning coefficient $\mathrm{k}$ for $\mathrm{Re}$ and $\mathrm{W}$ calculated from experiments and CALPHAD-simulations. $\widetilde{D}$ describing the effective interdiffusion coefficient at $1050{ }^{\circ} \mathrm{C}$.

\begin{tabular}{|c|c|c|c|c|c|c|}
\hline Alloy & \multicolumn{2}{|c|}{$\begin{array}{c}\text { Experiment } \\
\left(20^{\circ} \mathrm{C}\right)\end{array}$} & Ref & \multicolumn{3}{|c|}{$\begin{array}{c}\text { Simulation } \\
\left(1050^{\circ} \mathrm{C}\right)\end{array}$} \\
\hline & $k_{R e, \gamma / \gamma^{\prime}}$ & $k_{W, \gamma / \gamma^{\prime}}$ & & $k_{R e, \gamma / \gamma^{\prime}}$ & $k_{W, \gamma / \gamma^{\prime}}$ & $\begin{array}{c}\widetilde{D} \\
{\left[\mathrm{~m}^{2} / \mathrm{s}\right]}\end{array}$ \\
\hline CMSX-6 & n.a. & n.a. & - & n.a. & n.a. & $2,45 \mathrm{e}-15$ \\
\hline $\begin{array}{c}\text { M247LC- } \\
\text { SX }\end{array}$ & n.a. & 1,1 & {$[20]$} & n.a. & 1,4 & $1,9 \mathrm{e}-15$ \\
\hline LEK94 & n.a. & n.a. & - & 5,6 & 1 & $1,49 \mathrm{e}-15$ \\
\hline ReneN5 & 12,5 & 1,4 & {$[21]$} & 6,0 & 1,5 & $1,07 \mathrm{e}-15$ \\
\hline PWA1484 & 12,3 & 1,2 & {$[22]$} & 8,2 & 1,1 & $9,84 \mathrm{e}-16$ \\
\hline
\end{tabular}

Table 3: Comparison of diffusivities obtained from experiments $[23,24]$ and simulations, at $1200^{\circ} \mathrm{C}$.

\begin{tabular}{|c|c|c|c|c|c|c|c|c|c|}
\hline $\begin{array}{c}\mathrm{D} / 10^{-14} \\
{\left[\mathrm{~m}^{2} / \mathrm{s}\right]}\end{array}$ & $\mathrm{Al}$ & $\mathrm{Co}$ & $\mathrm{Cr}$ & $\mathrm{Mo}$ & $\mathrm{Ni}$ & $\mathrm{Re}$ & $\mathrm{Ta}$ & $\mathrm{Ti}$ & $\mathrm{W}$ \\
\hline Exp. & 11,1 & 1,9 & 2,8 & 1,1 & 2,2 & 0,07 & 2,7 & 7,2 & 0,3 \\
\hline Sim & 7,9 & 1,9 & 3,0 & 2,5 & 1,5 & 0,05 & 2,5 & 9,1 & 0,3 \\
\hline
\end{tabular}

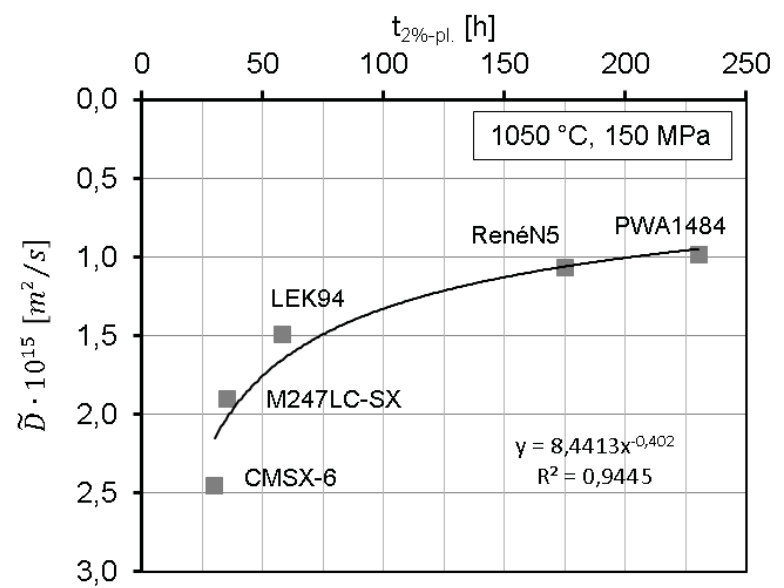

Figure 10. Correlation of the calculated effective interdiffusion coefficient $\widetilde{D}$ with the experimental creep life of the tested alloys.

In this study the effective diffusivity $\widetilde{D}$ was explicitly correlated with particle coarsening, which can lead to local penetration of $\gamma^{\prime}$-phase and thereby lead to additional plastic. This mechanism can be understood as softening of particle strengthening. Other works have argued however, that the reduction of the effective diffusivity primarily retards recovery mechanisms, see $[25,26]$. As the reduction of the effective diffusivity results from the substitution of Ni-matrix atoms with slow diffusing atom species, this can also be attributed to an optimisation of the $\gamma$-solid solution hardening. Reducing the creep strength optimisation of different 
superalloy generations onto a single mechanisms is therefore insufficient.

In consequence it is stated, that superalloys exhibit further optimisation potential by shifting especially the partitioning coefficient of tungsten $\left(k_{W, \gamma / \gamma^{\prime}}\right)$ or other slow diffusing refractory elements more strongly towards the $\gamma$-matrix.

Therefore the optimisation potential for $k_{W, \gamma / \gamma}$, was exemplarily estimated using a CALPHAD approach coupled with a statistical analysis. This approach is very cost and time effective, compared to a comprehensive experimental study of the solute element interdependencies. As in principle every solute element can affect the partitioning behaviour of tungsten, a permutation matrix was used in order to systematically calculate $k_{W, \gamma / \gamma}$. As a baseline the alloy LEK94 was chosen, on the grounds that this alloy contains a medium amount of rhenium in relation to the other alloys studied. From the baseline composition, elemental content was varied by \pm 1 at. $\%$ and the simulations were analysed with a linear regression model. The accuracy of this analysis is primarily dependent upon the accuracy of the thermodynamic database. The result for each calculation is the parameter $b_{i}$, which describes the change in the tungsten partitioning coefficient in relation to the content of respective solute element $i$. A positive value of $b_{i}$ then describes a redistribution of tungsten towards the matrix phase and the magnitude of $b_{i}$ allows a cross-comparison between the effectivity of alloying elements with regards to changing the partitioning ratio. Table 4 lists the first order interdependencies, these lie over an order of magnitude above the higher order interactions. The strongest influence can be attributed to the $\gamma^{\prime}$-forming elements $\mathrm{Al}, \mathrm{Ti}$ and $\mathrm{Ta}$ and when disregarding rhenium additionally Mo and $\mathrm{W}$ can exhibit a significant influence on the $k_{W, \gamma / \gamma}$, partitioning ratio.

Table 4: Regression coefficient of the primary interaction between element fraction and partitioning coefficient of tungsten.

\begin{tabular}{|c|c|c|c|c|c|c|c|c|}
\hline & $\mathrm{Al}$ & $\mathrm{Co}$ & $\mathrm{Cr}$ & $\mathrm{Mo}$ & $\mathrm{Re}$ & $\mathrm{Ta}$ & $\mathrm{Ti}$ & $\mathrm{W}$ \\
\hline $\mathrm{b}_{\mathrm{i}}$ & 0,11 & 0,01 & 0,07 & 0,10 & 0,12 & 0,06 & 0,10 & 0,08 \\
\hline
\end{tabular}

Figure 11 then illustrates the variation of the Mo-,Ti- and W-content in LEK94, where the colourscale reflects the change in $k_{W, \gamma / \gamma}$. In addition Figure 12-14 present comparable results for the other alloys beside CMSX-6, which does not contain tungsten as alloying element. All illustrations then reveal significant potential by systematic alloying for an improved $\mathrm{W}$-partitioning ratio. The partioning coefficient of tungsten as alloy design criteria alongside those identified by the established literature are studied with their interdependencies by Rettig et al. [27].

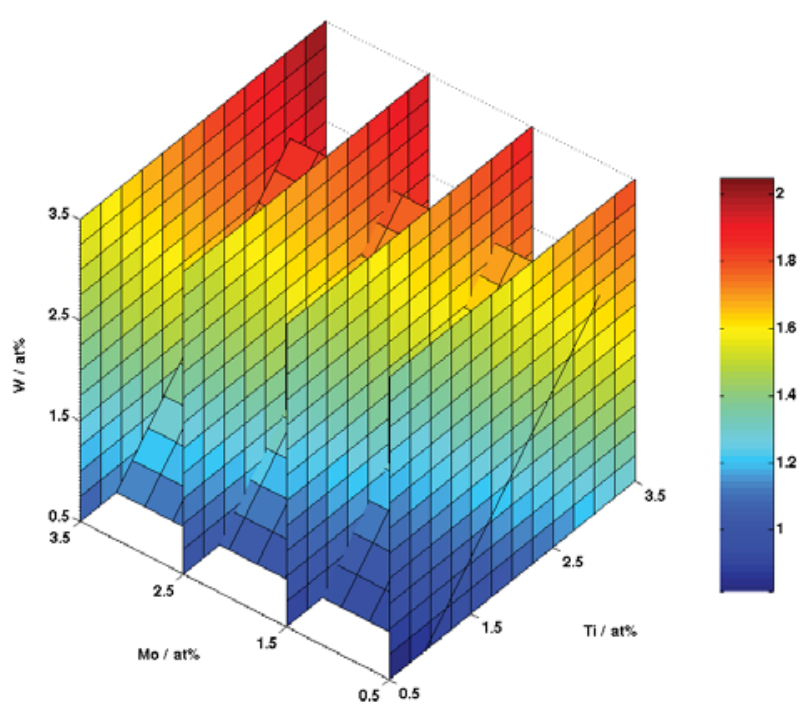

Figure 11: Dependence of the partitioning coefficient of tungsten in relation to a variation in Mo-, Ti-, and W-content [alloy: LEK94].

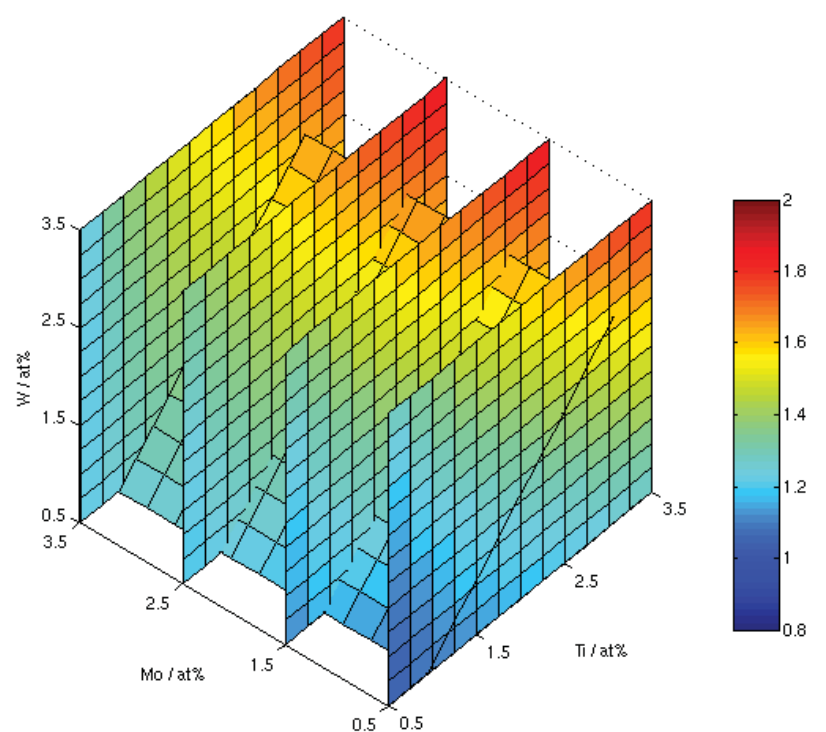

Figure 12: Dependence of the partitioning coefficient of tungsten in relation to a variation in $\mathrm{Mo-}$, Ti-, and $\mathrm{W}$-content [alloy: M247LC-SX]. 


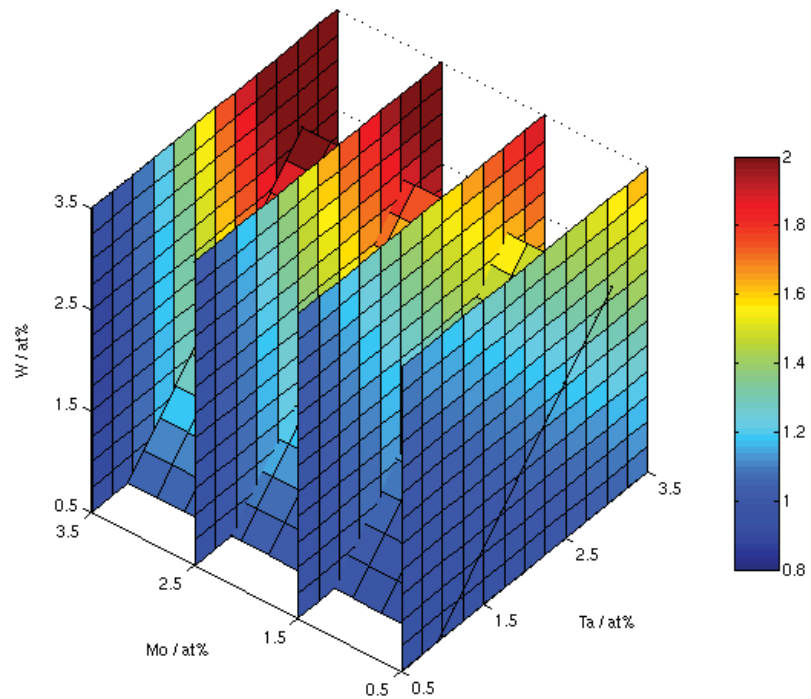

Figure 13: Dependence of the partitioning coefficient of tungsten in relation to a variation in Mo-, Ta-, and $\mathrm{W}$-content [alloy: ReneN5].

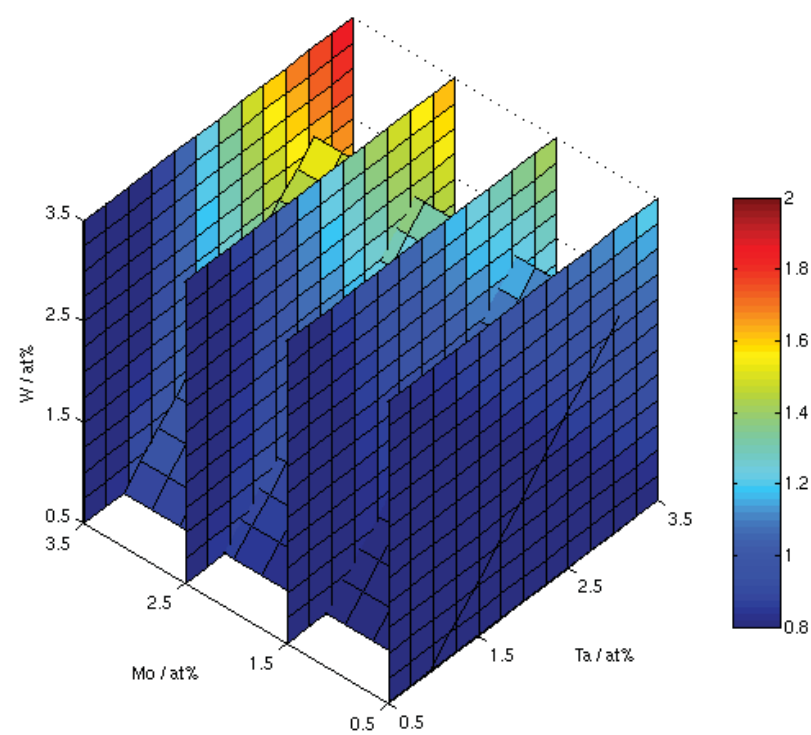

Figure 14: Dependence of the partitioning coefficient of tungsten in relation to a variation in $\mathrm{Mo}^{-}, \mathrm{Ta}-$, and $\mathrm{W}$-content [alloy: PWA1484].

\section{Discussion}

In general the simplified reproduction of the $\gamma / \gamma^{\prime}$-microstructure by a unit cell model appears to be insufficient to simulate creep deformation in the high temperature, low stress regime where rafting occurs.

The distribution of stresses in the matrix-phase as well as the equilibrium dislocation densities in matrix agree quantitatively with $[28,29]$, adding to the plausibility of the assumptions made in this model. Nevertheless the strain calculated by using the experimentally averaged microstructures, does not fit to the measured strain.
Svoboda models a unit cell of average cell dimensions that consistently increases in size. With the closure of vertical channels, recovery mechanisms by dislocation climb become inactive. Furthermore it is assumed that the threshold stress-state within a particle does not change with coarsening. However, when considering that coarsening results from a competitive growth process, leading to significant variations of $\gamma^{\prime}$-thickness, the latter assumptions cannot hold.

With the microstructural evolution the stress states in $\gamma$-channel and $\gamma^{\prime}$-precipitates change significantly. When considering nonaverage unit cell dimensions, local $\gamma / \gamma^{\prime}$-distributions can be calculated, where the critical resolved shear stress $\left(\tau_{\mathrm{PKO}}\right)$ is surpassed (see Figure 13).
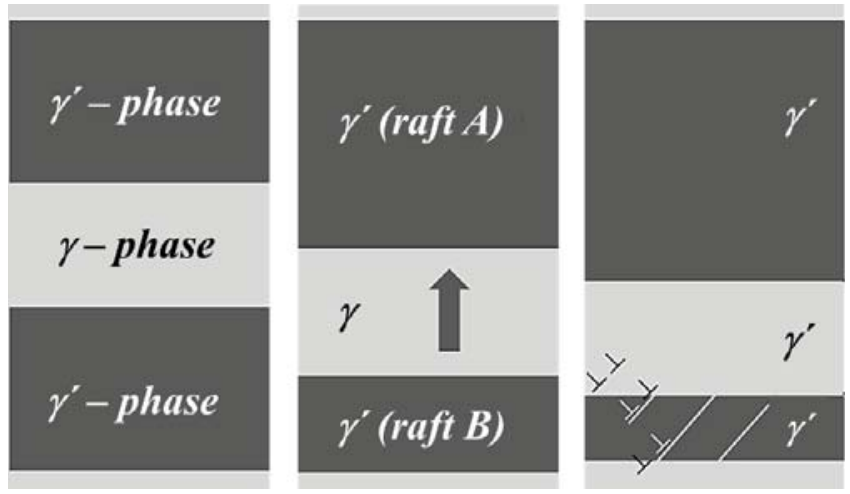

Figure 13: Schematic sketch of microstructure coarsening (from left to right) where due to competitive growth processes (reduction of interface energy at constant $\gamma^{\prime}$ volume fraction) and a rising thickness ratio, $\tau_{\mathrm{PK} 0}$ necessary for shearing is eventually surpassed (right).

This effect results from the crystal plasticity description in Svobodas model and can be summarised by the thickness ratiowhich describes the geometric ratio between $\gamma$-matrix and $\gamma^{\prime}$-precipitate necessary to surpass $\tau_{\mathrm{PK} 0}$ and lies for the alloy ReneN5 in the range of 2.5:1. The model however does not give an indication as to how substantial the additional plastic deformation by the precipitate phases is. Furthermore, the model assumes a constant value for the antiphase boundary energy. As the microstructure is alloy dependent, the same follows for the thickness ratio. SEM images from the interrupted creep tests then allow the identification of a number of areas above the thickness ratio ratio (see Figure 9).

Whilst individual shearing events depend on the local dislocation density in the matrix, the resulting back stress on the dislocation line (due to dislocation interactions and solid solution hardening), the repulsion forces on the $\gamma^{\prime}$-interphase from dislocation networks and the antiphase boundary energy, the thickness ratio emphasises the importance of a homogeneous microstructural evolution, by describing microstructural geometries, where particle shearing is likely to occur. Using a variety of different stress, temperature and alloy compositions, studies could show that the microstructural evolution contributes significantly to overall creep lifetime [30]. TEM studies have shown the increasing frequency of dislocation shearing events towards tertiary creep using comparable creep conditions testing the alloys LEK 94 and TMS-138 $[31,32,33]$. 
The speed of microstructural evolution is furthermore linked to the diffusion of solute elements through the matrix phase, which was shown to correlate well with the effective diffusion coefficient $(\widetilde{D})$ as described by [9]. The diffusion parameter $\widetilde{D}$ can then be significantly reduced with the addition of slow diffusion elements in the matrix, particularly by the slow diffusing, strongly $\gamma$-partitioning element rhenium. This agrees with phase field modelling results analysing the microstructural evolution [34]. As a replacement for rhenium with regards to the coarsening properties, tungsten has been found to display beneficial properties when the element partitioning coefficient is optimised in favour of the $\gamma$ matrix to ratios above 1.5 with the addition of titanium, molybdenum and tungsten. These CALPHAD-based trends agree with the experimental analysis by Heckl, where by modifying the alloy CMSX-4 into a titanium-free variant a reduction in the partioning of $\mathrm{Re}, \mathrm{W}, \mathrm{Mo}$ and $\mathrm{Cr}$ was observed [35]. However, the partitioning coefficient as well as the solid solution hardening cannot exceed the potential displayed per atom of rhenium added [36].

\section{Conclusions}

The comparison of experimental results using alloys of the first and second generation of superalloys with models using crystal plasticity and CALPHAD-approaches, have added to the understanding of the limitations contained in current models and allowed the identification of a number or optimisation potentials with regard to alloy design. In particular, the beneficial role of slow diffusing elements to the overall creep life has been discussed and interpretations by Fleischmann et al. [37] can be confirmed:

- Elements that have a low diffusivity and partition preferentially to $\gamma$-matrix, retard the increase of the strain rate

- The significant improvements in creep life, achieved by the addition of rhenium can be attributed to its low interdiffusivity in nickel alongside its low solubility in the $\gamma^{\prime}$-phase

In addition their effectivity is seen to not primarily arise from strengthening the matrix, but rather from their retardation of coarsening and stabilization of particle strengthening.

- Within the secondary creep stage local shearing of $\gamma^{\prime}$ becomes possible as a result of coarsened $\gamma^{\prime}$-rafts and increases the strain rate.

- Elements that have a low diffusivity and partition preferentially to $\gamma$-matrix retard particle coarsening

- A homogeneous distribution of $\gamma^{\prime}$-precipitates in the matrix with a unimodal size distribution decreases the likelihood of critical thickness ratios $\varphi_{\gamma / \gamma}$, developing in the early stages of creep

- Optimised partitioning coefficients, could further reduce the effective diffusivity within the $\gamma$-matrix. The proposed models have shown, that the addition of titanium and molybdenum are able to improve the partitioning of tungsten above a ratio of $1.5: 1$

Further experimental work is required in order to verify the optimisations predicted by the model and in order to establish this method as another alloy design criterion. Raising the effectiveness of tungsten could significantly increase price competitiveness, when considering cheaper rhenium-reduced alloys.

\section{Acknowledgments}

The authors acknowledge funding from the German Federal Ministry for Economic Affairs and Energy within the Federal Aeronautical Research Program "Lufo V/1". Lisa Koll and Hendrik Kramer (MTU Aero Engines AG) are gratefully acknowledged for generation of the SEM images. Shao Yan and Matthias Wagner (Technical University of Vienna) are gratefully acknowledged for their support with the MatCalc ${ }^{\circledR}-$ MATLAB $^{\circledR}$ interface. Erwin Povoden-Karadeniz (MatCalc Engineering $\mathrm{GmbH}$ ) is gratefully acknowledged for continuously improving me ni thermodynamic and mobility databases in parallel to this project.

\section{References}

[1] G. Petzow, "Metallographic Etching 2nd Edition", ASM International, Materials Park, United States of America, 1999, $124 f$.

[2] J. Svoboda, P. Lukáš, "Creep deformation modelling of superalloy single crystals", Acta Materialia, 48 (2000), 2519-2582.

[3] J. Svoboda, P. Lukáš, "Model of creep in< 001>-oriented superalloy single crystals", Acta Materialia, 46 (1998), 3421-3431.

[4] J. Svoboda, P. Lukáš, "Modelling of kinetics of direc-tional coarsening in Ni-superalloys", Acta Materialia. 44 (1996), 2557-2565.

[5] J. Svoboda, P. Lukáš, "Activation energy of creep in $<001>$ oriented superalloy CMSX-4 single crystals", Materials Science and Engineering: A. (1997), 173-176.

[6] J. Svoboda, P. Lukáš, "Modelling of recovery controlled creep in nickel-base superalloy single crystals", Acta Materialia, 45 (1997), 125-135.

[7] R.C. Reed, "The Superalloys - Fundamentals and Applications", Cambridge University Press, Cambridge, England, 2006.

[8] DE $10100790 \mathrm{C} 2$, „Nickel-Basislegierung für die gießtechnische Herstellung einkristallin erstarrter Bauteile", date of filing: 23.08.2003.

[9] C. Herring, "Diffusional Viscosity of a Polycrystalline Solid", Journal of Applied Physics, 21 (1950), 437-445.

[10] P. Caron, "High $\gamma$ ' Solvus New Generation Nickel-Based Superalloys for Single Crystal Turbine Blade Applications", in T. Pollock, R. Kissinger, R. Bowman, et al., eds. Seven Springs, USA: TMS, 2000), 737-746.

[11] N. Saunders, A. Miodownik, "CALPHAD (Calculation of Phase Diagrams): A Comprehensive Guide", Elsevier Science Ltd., Kidlington, 1998.

[12] E. Povoden-Karadeniz, "MatCalc thermodynamic and diffusion mobility databases for Ni-base superalloys". www.matcalc.tuwien.ac.at, 2016. 
[13] Göhler, T., "Designkriterien für rheniumreduzierte Nickelbasis-Einkristalllegierungen: Identifikation und rechnergestützte Umsetzung", $\mathrm{PhD}$ Thesis, University ErlangenNürnberg, 2016, to be published.

[14] Scheffler, E., „Statistische Versuchsplanung und auswertung. Eine Einführung für Praktiker“", 3. Auflage, Dt. Verlag für Grundstoffindustrie, Stuttgart, 1997.

[15] C.W.M. Schwalbe, „Verifizierung des Kriechmodells nach Svoboda und Lukas im Hinblick auf die Vergroeberungsdynamik von einkristallinen Nickelbasissuperlegierungen der ersten und zweiten Generation", master thesis, TU Berlin, 2014.

[16] B. Reppich, "Some new aspects concerning particle hardening mechanism in $\gamma^{6}$ precipitating Ni-Base alloys - I Theoretical concept", Acta Metallurgica, 30 (1982), 87-94.

[17] A. Kruk, B. Dubiel, A. Czyrska-Filemonowicz, „The 3D imaging and metrology of CMSX-4 superalloy microstructure using FIB-SEM tomography method", Solid State Phenomena, 197 (2013), 89-94.

[18] W. Chen, J.P. Immarigeon, "Thickening behaviour of $\gamma^{\prime}$ precipitates in nickel base superalloys during rafting", Scripta materialia, 39 (1998), 167-174.

[19] Biermann, H., "Ursachen und Auswirkungen der gerichteten Vergröberung („Floßbildung“) in einkristallinen Nickelbasis-Superlegierungen”, VDI Verlag, Düsseldorf, 1999.

[20] H. Harada, K. Ohno, T. Yamagata, "Phase calcula-tion and its use in alloy design program for nickel-base superalloys", in S. Reichman, D.N. Duhl, S. Antolovich, C. Lund, eds., (Seven Springs, USA: TMS, 1988), 733-742.

[21] T.M. Pollock, R.D. Field, "Dislocations and HighTemperature Plastic Deformation of Superalloy Single Crystals", in F.R.N. Nabarro, M.S. Duesbery, eds., Dislocations in Solids, Elsevier, 11 (2002).

[22] M.K. Miller, L.S. Lin, A.D. Cetel, H. Harada, H. Murakami, "Partitioning Behavior of Alloying Elements in PWA1484", in Proceedings Microscopy and Microanalysis, Michigan, Minneapolis, 1996.

[23] M. Karunaratne, D. Cox, P. Carter, R. Reed, "Modelling of the microsegregation in CMSX-4 superalloy and its homogenisation during heat treatment", in T. Pollock, R. Kissinger, R. Bowman, et al., eds., (Seven Springs, USA: TMS, 2000), 263-272.

[24] M. Karunaratne, P. Carter, R. Reed, "Interdiffusion in the face-centred cubic phase of the Ni-Re, Ni-Ta and Ni-W systems between 900 and $1300{ }^{\circ} \mathrm{C}$ ", Materials Science and Engineering: A, 281 (2000), 229-233.

[25] Johnson, W.R.; Barrett, C.R.; Nix, W.D., "The high- temperature creep behavior of nickel-rich Ni-W solid solutions", Metallurgical and Materials Transactions, 3 (1972), S. 963-969.
[26] Z. Zhu, H. Basoalto, N. Warnken, R.C. Reed, "A model for the creep deformation behaviour of nickel-based single crystal superalloys", Acta Materialia, 60 (2012), 4888-4900.

[27] R. Rettig, N.C. Ritter, A. Müller, K. Matuszewsja, R.F. Singer, „Development of a low-density rhenium-free single crystal nickel-based superalloy by application of numerical multi-criteria optimization using thermodynamic calculations", (Seven Springs, USA: TMS, 2016), to be published.

[28] L. Müller, U. Glatzel, M. Feller-Kniepmeier, "Modelling thermal misfit stresses in nickel-base superalloys containing high volume fraction of $\gamma^{\prime}$ - phase", Acta Metallurgica et Materialia, 40 (1992), 1321-1327.

[29] H. Harada, Y. Gu, "High Temperature Materials Researches for Gas Turbine and Aeroengines at NIMS", 4th WMRIF, IMR, Shenyang, 2011.

[30] U. Tetzlaff, H. Mughrabi, "Enhancement of the hightemperature tensile creep strength of monocrystalline nickel-base superalloys by pre-rafting in compression", ", in T. Pollock, R. Kissinger, R. Bowman, et al., eds., (Seven Springs, USA: TMS, 2000), 273-282.

[31] L.A. Jácome, P. Nörtershäuser, J.K. Heyer, A. Lahni, "High-temperature and low-stress creep anisotropy of single-crystal superalloys", Acta Materialia, 61 (2013), 29262943.

[32] L.A. Jácome, P. Nörtershäuser, C. Somsen, "On the nature of $\gamma^{\prime}$ phase cutting and its effect on high temperature and low stress creep anisotropy of Ni-base single crystal superalloys", Acta Materialia, 69 (2014), 246-264.

[33] J.X. Zhang, T. Murakumo, H. Harada, Y. Koizumi, "Dependence of creep strength on the interfacial dislo-cations in a fourth generation SC superalloy TMS-138", Scripta Materialia, 48 (2003), 287-293.

[34] J. Kundin, L. Mushongera, T. Goehler, H. Emmerich, "Phase-field modeling of the $\gamma^{\prime}$-coarsening behavior in Nibased superalloys", Acta Materialia, 60 (2012), 3758-3772.

[35] Heckl, A., Auswirkungen von rhenium und Ruthenium auf die Mikrostruktur und Hochtemperaturfestigkeit von NickelBasis Superlegierungen unter Berücksichti-gung der Phasenstabilität, $\mathrm{PhD}$ Thesis, University Erlangen-Nürnberg, 2011.

[36] E. Fleischmann, M.K. Miller, E. Affeldt, U. Glatzel, “Quantitative experimental determination of the solid solution hardening potential of rhenium, tungsten and molybdenum in single-crystal nickel-based superalloys", Acta Materialia, 87 (2015), 350-356.

[37] E. Fleischmann, C. Konrad, J. Preußner, R. Völkl, U. Glatzel, "Influence of Solid Solution Hardening on Creep Properties of Single-Crystal Nickel-Based Superalloys", Metallurgical and Materials Transactions, 46A (2015), 11251130. 


\section{Appendix A}

Nomenclature

b burgers vector

$b_{i} \quad$ regression coefficient from statistical analysis of primary interaction between property and influencing variable (i)

$D_{i}^{\gamma} \quad$ diffusivity of chemical element $\mathrm{i}$ in $\gamma$-phase at respective temperature

$\widetilde{D} \quad$ effective interdiffusion coefficient

G shear modulus

$h_{x}, h_{y}, h_{z} \quad$ thickness of the $\gamma$-channels in respective direction

$\mathrm{L}_{\mathrm{x}}, \mathrm{L}_{\mathrm{y}}, \mathrm{L}_{\mathrm{z}} \quad$ dimensions of the $\gamma^{\prime}$-particle

$\mathrm{k}_{\mathrm{x}, \gamma / \gamma^{\prime}} \quad$ partioning coefficient of element $\mathrm{x}$ between $\gamma$ -

matrix and $\gamma^{\prime}$-particle

$\mathrm{t} \quad$ time

w material specific constant for the calculation of $\tau_{\mathrm{PK} 0}$

$X_{i}^{\gamma} \quad$ content of chemical element $\mathrm{i}$ in $\gamma$-phase at respective temperature

$\gamma \quad$ gamma matrix phase

$\gamma^{\prime} \quad$ gamma prime phase

$\gamma_{\mathrm{APB}} \quad$ antiphase boundary energy

$\varepsilon \quad$ plastic strain

$\tau_{\mathrm{P}} \quad$ resolved shear stress for cutting of $\gamma^{\prime}$-particle

$\tau_{\mathrm{P} 1}, \tau_{\mathrm{P} 2}, \tau_{\mathrm{P} 3}$ resolved shear stress in $\gamma^{\prime}$-particle for glide system groups defined by four equivalent slip planes

$\tau_{\text {PK0 }} \quad$ threshold shear stress for cutting of $\gamma^{\prime}$-particle

$\tau_{\mathrm{y}} \quad$ resolved shear stress for slip in horizontal $\gamma$-channels

$\tau_{\mathrm{y} 1}, \tau_{\mathrm{y} 2}, \tau_{\mathrm{y} 3} \quad$ resolved shear stress in horizontal $\gamma$-channels defined by glide system groups, each containing four equivalent slip planes

$\tau_{\mathrm{y} 0} \quad$ threshold shear stress for slip in horizontal $\gamma$-channels

$\varphi_{\gamma / \gamma^{\prime}} \quad \gamma$-channel to $\gamma^{\prime}$-particle thickness ratio 